\title{
The theoretical influence of the difference between the LUMO energy levels of donor and acceptor in organic photovoltaic triple-junction solar cells
}

\section{Ben Minnaert $^{1, *}$, Tom Vandecaveye ${ }^{1}$ and Peter Veelaert ${ }^{1}$}

1 Faculty of Engineering and Architecture, Ghent University, Valentin Vaerwyckweg 1, B-9000 Gent, Belgium; E-Mails: Ben.Minnaert@UGent.be (B.M.); Tom.Vandecaveye@ugent.be (T.V); Peter.Veelaert@UGent.be (P.V.)

* Author to whom correspondence should be addressed; E-Mail: Ben.Minnaert@UGent.be (B.M.); Tel.: +32-9-243-2589. Received: 31 March 2014 / Accepted: 15 May 2014 / Published: 26 May 2014

\begin{abstract}
In organic photovoltaic solar cells, light absorption does not immediately lead to free charge carriers. Instead, an exciton is created. The highest efficiency is reached when the lowest unoccupied molecular orbital (LUMO) of the donor is as close as possible to the LUMO of the a cceptor. However, a n ecessary condition for efficient dissociation of the created excitons is that the difference between the LUMOs of donor and acceptor is higher than the exciton binding energy. The value of the exciton binding energy in $\mathrm{d}$ ifferent materials is a subject of discussion. The excess of this necessary minimum of the LUMOdifference corresponds with an energy loss. Moreover, it is often not possible to optimize suitable material combinations for or ganic photovoltaic cells to an i deal (low) LUMO difference. Another energy loss in organic solar cells is caused by their narrow absorption windows, compared to the absorption band of in organic solar cells. A way to capture a wider band of the solar radiation is using more solar cells with different bandgaps in a row. In this article, we study three organic cells in a row, i.e. a triple-junction. More specifically, we study the the oretical influence of the difference between the LUMO energy levels of donor and acceptor for a $\mathrm{n}$ organic triple-junction solar cell. We study as well the monolithic as the stacked configuration.
\end{abstract}

Keywords: solar cells; ph otovoltaic energy; triple-junction; organic solar cells; multijunction; simulation; energy levels; lowest unoccupied molecular orbital; LUMO 


\section{Introduction}

A characteristic of organic solar cells is th eir narrow absorption $\mathrm{w}$ indow, compared to the absorption band of inorganic sem iconductors [1]. A possible way to capture a wider band of the solar spectrum - and thus in creasing the power con version efficiency - is using $\mathrm{m}$ ore solar cells with different bandgaps in a row, referred to as a $\mathrm{m}$ ulti-junction solar cell. In this article, we will focus on triple-junction solar cells, i.e. three cells in a row. The absorber of the first single solar cell in such a triple-junction cell has a large bandgap $E_{g l}$. High-energy photons with an energy $h v>E_{g l}$ are absorbed by the first cell. The second cell, with a lower bandgap $E_{g 2}$, absorbs the middle-energy photons with energy between $E_{g 1}$ and $E_{g 2}$. The third cell absorbs the low-energy photons between $E_{g 2}$ and $E_{g 3}$ (Figure 1). In this configurati on, the photon energy is used $\mathrm{m}$ ore efficiently: the voltage at which electrical charge is collected in each subcell is closer to the energy of the photons absorbed in that subcell.

Figure 1. (a) A stacked or 6-terminal triple-junction solar cell: the first single cell absorbs photons with energy higher than $E_{g 1}$. The second and third cell absorb photons with energy between $E_{g 1}$ and $E_{g 2}$, respectively, $E_{g 2}$ and $E_{g 3}$. Photons with energy below $E_{g 3}$ are not absorbed. The three su bcells are electrically s eparated. (b) A m onolithic or 2-terminal triple-junction solar cell: the single cells are electrically connected in series.

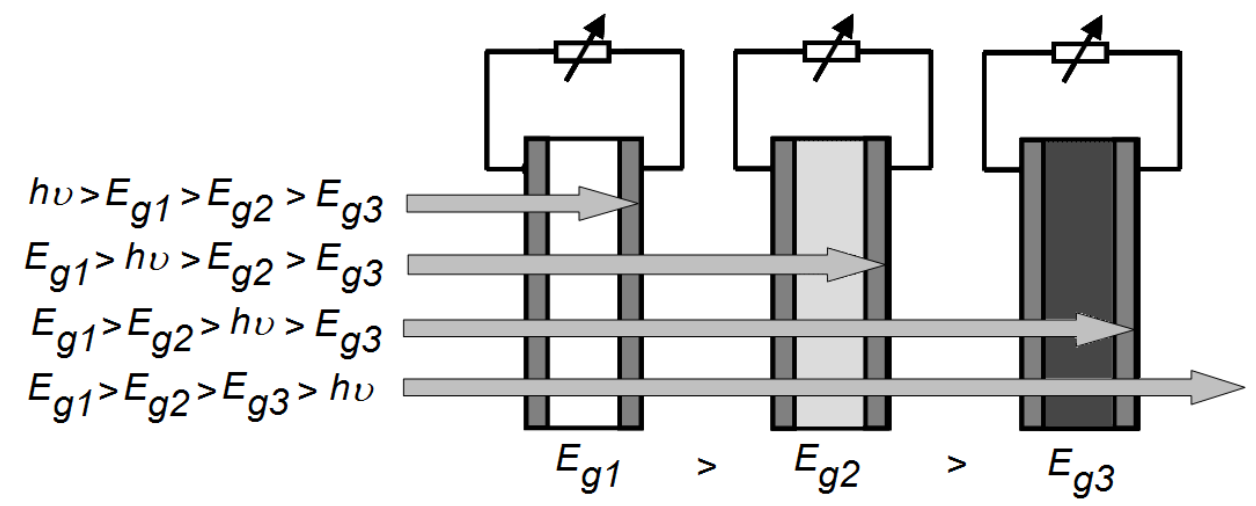

(a)

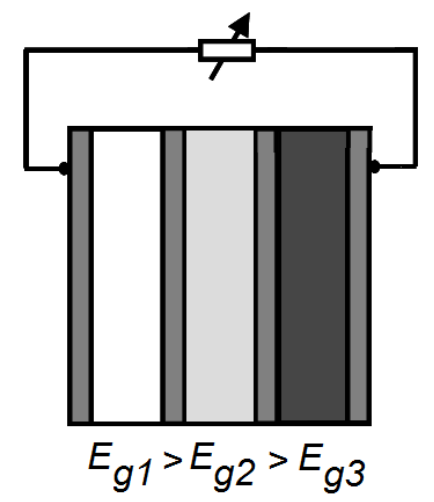

(b)

In the ideal configuration, the subc ells are electrically separated. This is called the stacked or 6terminal configuration (Figure 1a). However, experimental and commercial multi-junction solar cells are usually of the monolithic type (Figure 1b). This means that they are not only optically in series, but also electrically in series. This configuration will never reach an efficiency that is higher than that of a stacked (6-terminal) triple-junction cell, because all single cells cannot be operating at their optim al working point at the same time (unless they have an equal maximum-power current).

\section{Assumptions}

The active material in a single organic bulk heteroj unction solar cell consists of an interpenetrating network of an electron accepto $r$ (e.g. fullerene de rivatives) and an electron dono $r$ (e.g. conjugated polymers), sandwiched between two electrodes with different work functions. The optical bandgap $E_{g}$ 
is defined as the difference between the lowest $\mathrm{u}$ noccupied molecular orbital (LUMO) and the highest occupied molecular orbital (HOMO) of the absorber material.

We consider a 6 -terminal triple-junction solar cell, consisting of three single organic cells. We assume that in each sing le cell, only one m aterial absorbs light. Usually, most of the light is abso rbed by the donor; this is the case we will con sider here onwards. Because we assum e full absorption in each subcell, we neglect interference and optical coupling of the subcells, thu s overestimating the efficiency potential. The organic cell with the widest absorber bandgap is at top (at the side of the sun), thus $E_{g 1}>E_{g 2}>E_{g 3}$. The distance between the HOMO of the donor and the LUMO of acceptor is considered as the therm odynamic limitation of the useful energy [2]. We call this value the interf ace bandgap $E_{i}$. For an organic solar cell with ohm ic contacts, the ope n circuit voltage $V_{o c}$ is linearly dependent on the interface bandgap $E_{i}$. For a cell with non-ohmic contacts, the $V_{o c}$ is dependent on the work function difference of the electrodes. In these calculations, we assume a cell with ohmic contacts.

For our simulation, the following funda mental assumptions are m ade about the stacked triplejunction cell (Figure 1a): (i) every photon with energy $h v$ higher than the bandgap $E_{g l}$ is absorbed by the first cell and leads to a useful energy $E_{i l}$. This assumption im plies that each absorbed photon eventually leads to a free electron and a free hole, w ith an energy difference of $E_{i l}$ between them. (ii) every photon with energy $h v$ between $E_{g 1}$ and $E_{g 2}$ is absorbed by the second cell and leads to a useful energy $E_{i 2}$. (iii) every photon with energy $h v$ between $E_{g 2}$ and $E_{g 3}$ is absorbed by the th ird cell and leads to a u seful energy $E_{i 3}$. (iii) photons with energy $h v$ lower than $E_{g 3}$ are fully transmitted. The maximum efficiency $\eta_{\max }$ is therefore given by:

$$
\eta_{\max }=\frac{E_{i 1} \int_{E_{g 1}}^{\infty} N(E) d E+E_{i 2} \int_{E_{g 2}}^{E_{g 1}} N(E) d E+E_{i 3} \int_{E_{g 3}}^{E_{g 2}} N(E) d E}{\int_{0}^{\infty} E N(E) d E}, \text { with } E_{g 1}>E_{g 2}>E_{g 3}
$$

with $N(E)$ the incident photon flux. For all our sim ulations, we use the AM 1.5 experimentally measured solar spectrum [3]. In this ideal s cenario, the open circuit voltage $V_{o c}$ of the subcells will be given by $E_{i j} / q(j=1,2,3)$ with $q$ the electric charge. The fill factor $F F$ of the subcells is assumed to equal unity, as well as the ex ternal quantum efficiency $E Q E$ of the first cell for wavelengths below the cutoff wavelength $\lambda_{\mathrm{g} 1}$ (corresponding with $E_{g l}$ ). Similar conditions apply to the second and third cell.

In a monolithic triple-junction solar cell (Figure 1b), the individual cells are electrically connected in series. This means that the total voltage over the cell is the sum of the voltages over each indiv idual cell, and thus equals the sum of the interface ba ndgaps of the single cells. Furtherm ore, the same current flows through all single cells. Hence, the m aximum efficiency $\eta_{\max }$ for a monolithic organic triple-junction cell is given by:

$$
\eta_{\max }=\frac{\left(E_{i 1}+E_{i 2}+E_{i 3}\right) \cdot \min \left(\int_{E_{g 1}}^{\infty} N(E) d E, \int_{E_{g 2}}^{E_{g 1}} N(E) d E, \int_{E_{g 3}}^{E_{g 2}} N(E) d E\right)}{\int_{0}^{\infty} E N(E) d E}, \text { with } E_{g 1}>E_{g 2}>E_{g 3}
$$


with $\min (x, y, z)$ the minimum of $x, y$ and $z$. The open circuit voltage $V_{o c}$ of the whole monolithic cell will be giv en by $\left(E_{i 1}+E_{i 2}+E_{i 3}\right) / q$, the fill factor $F F$ equals unity, as does the external quantum efficiency $E Q E$ for wavelengths below the cut-off wavelength.

In organic bulk heterojunction solar cells, light absorption does not immediately lead to free charge carriers. Instead, an exciton is created. In an ideal scenario, the highest efficiency is reached when the LUMO of the donor is as close as possible to the LUMO of th e acceptor. However, a necessary condition for efficient dissociation of the created excitons is that the difference between the LUMOs of donor and acceptor ( $\triangle \mathrm{LUMO})$ is higher than th exciton binding energy [4]. The value of the exciton binding energy (and the minimal $\triangle \mathrm{LUMO}$ ) in different materials is a subject of discussion, but a value of $0.3 \mathrm{eV}$ was put forward as an empirical threshold necessary for exciton dissociation [5]. The excess of this necessary minimum of the LUMO-difference corresponds with an energy loss.

In the next section, we calcu late the theoretical influence of the diff erence between the LUMO energy levels of donor and accep tor for an org anic stacked and monolithic triple-junction solar cell. The absolute value of the maximum efficiency is only relevant for academic purposes. It is the relative difference between the efficiencies that is impor tant in quantif ying the inf luence of the LUMO differences.

\section{Results}

To study the influence of $\triangle \mathrm{LUMO}$, we calculate the m aximum efficiency in this ideal scenario by changing this parameter, and determ ine for each $\triangle \mathrm{LUMO}$ the optim al bandgaps for the different subcells. First, we only change $\Delta \mathrm{LUMO}_{1}$ (the $\Delta \mathrm{LUMO}$ of the firs $\mathrm{t}$ subcell) and keep $\Delta \mathrm{LUMO}_{2}$ and $\Delta \mathrm{LUMO}_{3}$ constant at $0.3 \mathrm{eV}$ (the empi rical threshold necessary for exciton dissociation). If there is no energy difference between the LUMOs of the first s ubcell, the maximum efficiency reaches $62 \%$ and $61 \%$ for a stacked and monolithic configuration respectively (Figure $2 \mathrm{a}$ ). The efficiency at $\Delta \mathrm{LUMO}_{1}=$ $0.3 \mathrm{eV}$, the m inimum threshold for exciton dissociati on, is $56 \%$ and $55 \%$ respect ively, a decrease of $10 \%$ relative compared to no LUMO difference. The e fficiency decreases 1 to $3 \%$ relative per $0.1 \mathrm{eV}$. This relative decrease is higher for lower values of $\Delta \mathrm{LUMO}_{1}$. The optimal bandgap $E_{g l}$ increases with increasing $\triangle \mathrm{LUMO}_{1}$ for both the stacked and the monolithic configuration. The higher the LUMO difference, the smaller the part of the incom ing spectrum that is bein $g$ absorbed. This reduces the relative decrease per $0.1 \mathrm{eV}$. The op timum of all three b andgaps increase with higher $\Delta \mathrm{LUMO}_{1}$. This was to be expected. Ind eed, a high $\Delta \mathrm{LUMO}_{1}$ of the f irst subcell will lo wer significantly the u seful energy of the absorbed photons in this first subcell. This is compensated by increasing $E_{g l}$. As a result, a broader part of the solar spectrum is transmitted to the other two subcells, leading to a rearrangement of the optim al bandgaps of those subcells to hi gher values. The maxim um efficiency will never decrease below $49.5 \%$, because this is the efficien cy of a ta ndem cell (i.e. a $m$ ulti-junction with two subcells) where both $\triangle \mathrm{LUMOs}$ are $0.3 \mathrm{eV}$. The bandgap of the first solar cell will then be that big that it will no longer absorb any photons and the triple-junction will act as a tandem cell.

We now consider the influence of $\Delta \mathrm{LUMO}_{2}$ (with $\Delta \mathrm{LUMO}_{1}=\Delta \mathrm{LUMO}_{3}=0.3 \mathrm{eV}$ ). The efficiency drops from $64 \% / 61 \%$ at $0 \mathrm{eV}$ to $56 \% / 55 \%$ for $0.3 \mathrm{eV}$ and $50 \% / 43 \%$ for $1.0 \mathrm{e} \mathrm{V}$ for the stacked / monolithic configuration respectively (Figure $2 \mathrm{~b}$ ). We notice a sharp decline in the beginning which decreases for higher $\Delta \mathrm{LUMO}_{2}$ values. The explanation is analogous as for

$\Delta \mathrm{LUMO}_{1}$. For higher 
$\triangle \mathrm{LUMO}_{2}$ values, this decrease dim inishes fast. Analogous conclusions as for $\Delta \mathrm{LUMO}_{1}$ can be drawn for the optimal bandgaps: the ideal bandgap of the second subcell in creases with higher $\Delta \mathrm{LUMO}_{2}$ values to compensate for the en ergy loss caus ed by the LUMO difference. As a result, the o ptimal bandgap of the first subcell decreases whereas $E_{g 3}$ increases. This reduces the influence of the second (less efficient) subcell. At high $\Delta \mathrm{LUMO}_{2}$ values, the optimal values of $E_{g 1}$ and $E_{g 2}$ coincide, reducing the triple junction to a tandem cell. Analogous conclusions can be drawn for $\Delta \mathrm{LUMO}_{3}$ (Figure $2 \mathrm{c}$ ).

Figure 2. (left axis) The maximum efficiency for a stacked (solid line) and monolithic (dashed line) triple-junction solar cell as function of (a) $\Delta \mathrm{LUMO}_{1}$, (b) $\Delta \mathrm{LUMO}_{2}$ and (c) $\triangle \mathrm{LUMO}_{3}$. (right axis) The optim al bandgaps of the th ree subcells as function of (a) $\Delta \mathrm{LUMO}_{1}$, (b) $\Delta \mathrm{LUMO}_{2}$ and (c) $\Delta \mathrm{LUMO}_{3}$ for a stacked (filled symbols) and monolithic (open symbols) triple-junction solar cell.
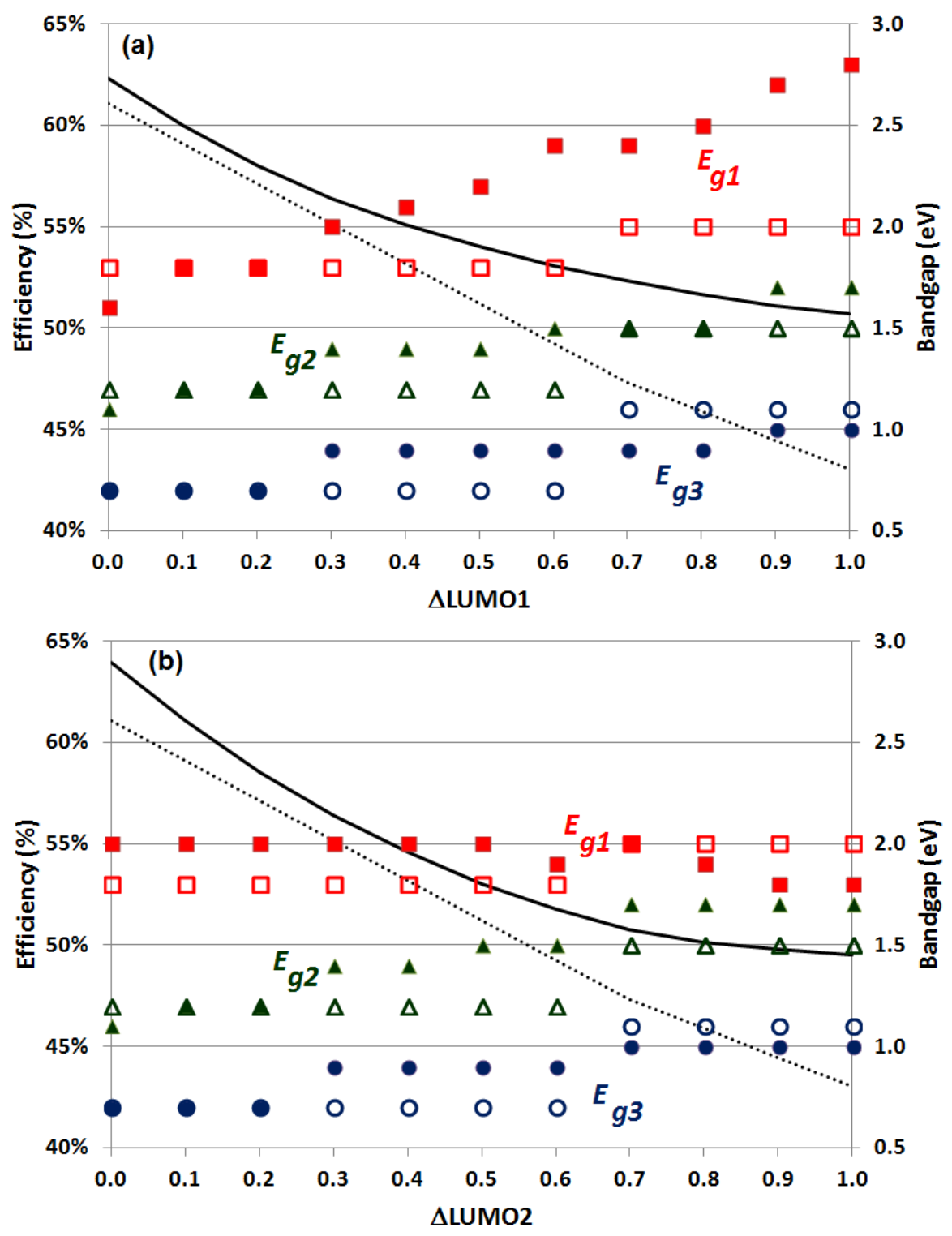


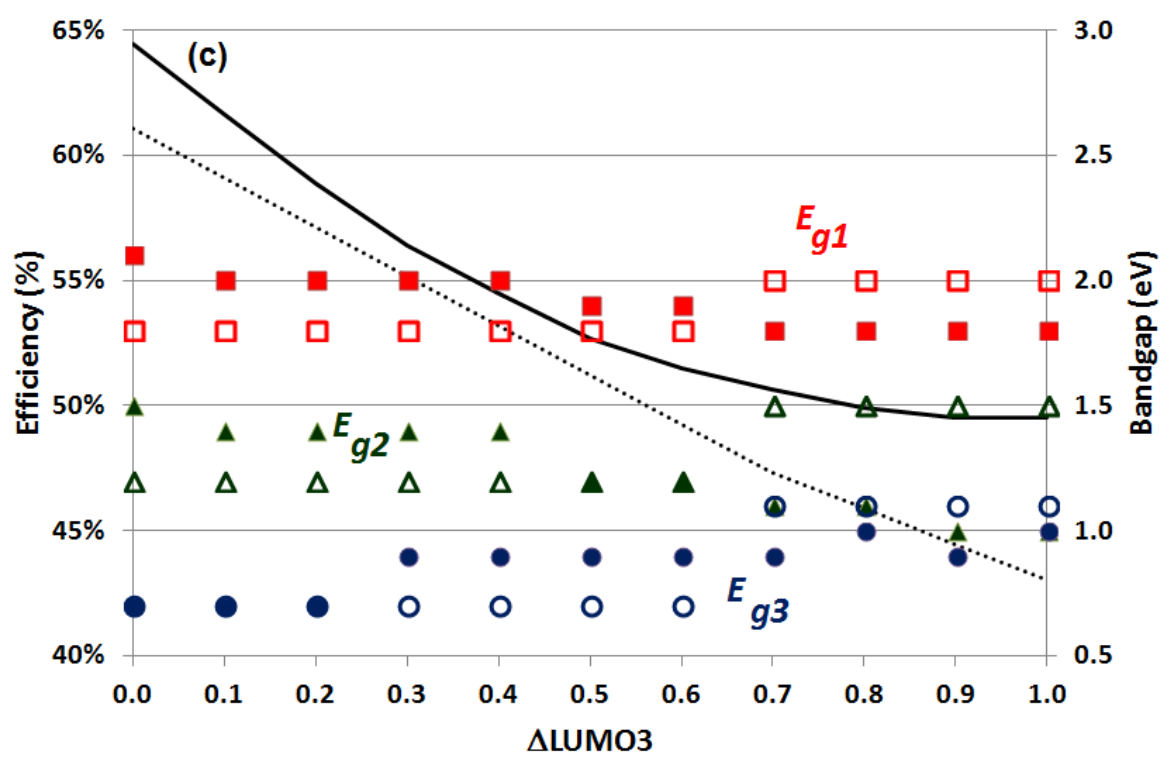

\section{Conclusions}

The most important conclusion fr om this study is that a high $\quad \Delta$ LUMO for one subcell is not detrimental for the efficiency of an organic triple-junction solar cell. It is even of ten better to combine two subcells with low $\triangle$ LUMOs with one subcell with a h igh $\triangle \mathrm{LUMO}$, than combining three subcells with average $\triangle$ LUMOs. This conclusion follows from the increasingly smaller decrease in efficiency with increasing $\triangle$ LUMOs.

\section{Conflicts of Interest}

The authors declare no conflict of interest.

\section{References and Notes}

1. Winder, C.; Sariciftci, N.S., Low bandgap polymers for photon harvesting in bulk heterojunction solar cells. J. Mater. Chem. 2004, 14, 1077-1086.

2. Dennler, G.; Sariciftci, N.S., Flexible conjugated polym er-based plastic solar cells: From basics to applications. Proc. IEEE. 2005, 93, 1429-1439.

3. International Standard, IEC 60904-3, Edition 2, April 2008, Photovoltaic devices - Part 3 : measurement principles for terr estrial photovoltaic (PV) solar de vices with refe rence spectral irradiance data, ISBN 2-8318-9705-X, International Electrotechnical Commission (2008).

4. Sun, S-S.; Optimal energy offsets for orga nic solar cells containing a donor/acceptor pair. Sol. Energy Mater. Solar Cells 2005, 85, 261-267.

5. Scharber, M.C.; Mühlbacher, D.; Koppe, M.; Denk, P.; Waldauf, C.; Heeger, A.J.; Brabec, C.J., Design rules for donors in bulk -heterojunction solar ce lls - Towards $10 \%$ energy -conversion efficiency. Adv. Mat. 2006, 18, 789-794.

(C) 2014 by the au thors; licensee MDPI, Basel, Switz erland. This article is an open access article distributed under the term $\mathrm{s}$ and condition $\mathrm{s}$ of the Creativ e Commons Attribution license (http://creativecommons.org/licenses/by/3.0/). 